\title{
The Clinical Effects of Hyperbaric Oxygen in the Treatment of Sudden Deafness
}

\author{
Xinli Feng1, Ying Chang2* \\ ${ }^{1}$ Hyperbaric Oxygen Treatment Room, Affiliated Hospital of Hebei University, Baoding 071000, Hebei Province, China \\ ${ }^{2}$ Department of Gastroenterology, Affiliated Hospital of Hebei University, Baoding 071000, Hebei Province, China
}

*Corresponding author: Ying Chang, chyi4815@126.com

\begin{abstract}
Objective: To observe the clinical effect of hyperbaric oxygen in the treatment of sudden deafness. Methods: Ninety-six patients with sudden deafness diagnosed by the otolaryngology department were divided into 2 groups which comprised of 48 patients in the conventional treatment group and the other 48 patients in the hyperbaric oxygen treatment group. Both groups were treated with methylcobalamin, vitamin B1, and ginkgo biloba extract. The patients in the hyperbaric oxygen treatment group were given hyperbaric oxygen therapy of 2.0 ATA once a day. Each course of treatment lasted 10 days and after two courses of treatment, the clinical efficacies of the two groups were compared. Results: After two courses of treatment, the effect of treatment in the hyperbaric oxygen treatment group was significantly better than that of the conventional treatment group. Conclusion: Hyperbaric oxygen therapy can effectively improve the hearing level of patients with sudden deafness and the overall effective rate of treatment.
\end{abstract}

Keywords: Sudden deafness; Hyperbaric oxygen; Clinical effect

Publication date: July 2021; Online publication: July 31, 2021

\section{Introduction}

Sudden sensorineural hearing loss (SSHL) refers to sensorineural hearing loss of unknown cause that occurs within 72 hours. It is a common and frequently occurring disease in clinical otolaryngology with an incidence rate of 10 to 100,000 and a year by year increasing trend ${ }^{[1-2]}$. Clinically, it is more common for unexplained sensory hearing loss. Hearing drops sharply within a few minutes to three days after the onset of the disease and that is when the disease reaches its peak. Patients are usually admitted to the hospital with unilateral deafness. They may develop deafness, dizziness, nausea, and vomiting before or after the onset. The disease can be seen in all age groups but most of the patients are from the middle-aged and the elderly group. The etiology of sudden deafness has not been fully elucidated. Previous reports included viral infections, vascular occlusions, damage to the labyrinth window membrane, autoimmune diseases, and abnormal cellular stress responses ${ }^{[3-4]}$. Clinically, the principle of treatment in regard to SSHL is early detection, early diagnosis, and early treatment. The main treatment methods include drugs, hyperbaric oxygen, and surgery. Hyperbaric oxygen (HBO) is a common method for the treatment of sudden sensorineural hearing loss and it is used in combination with drug therapy to improve the curative effect. HBO refers to the inhalation of pure oxygen or high-concentration oxygen in an environment with more than one standard atmospheric pressure which rapidly increases human blood oxygen content, blood oxygen tension, and blood oxygen diffusion distance in which the amount of dissolved oxygen in the blood rises from $0.3 \%$ of normal pressure to $5.3 \%$ and the diffusion distance of oxygen increases from $30 \mathrm{~mm}$ under normal pressure to $100 \mathrm{~mm}$, thereby correcting tissue hypoxia ${ }^{[5]}$. The patients with sudden deafness that were admitted to the Affiliated Hospital of Hebei University in the past three years were grouped in order 
to analyze the effects of different treatment methods.

\section{Materials and methods}

\subsection{Clinical data}

A total of 96 patients with sudden deafness who were treated by the hospital's otolaryngology department from February 2018 to February 2021 were selected in which all the patients had met the diagnostic criteria for sudden deafness set by the Chinese Otolaryngology Society in the year 2000. Using the random number table method, the patients were divided into 2 groups which were the hyperbaric oxygen treatment group and the conventional treatment group. The patients that were selected comprised of 55 males and 41 females with age ranging from 13 to 72 years old in which the average age was 41.2 years old. The patients were admitted to the hospital within 1 week of the onset. The differences in the general data such as gender, age, time of onset, etc. were not statistically significant $(\mathrm{P}>0.05)$ and they were comparable.

\subsection{Inclusion and exclusion criteria}

The inclusion criteria were patients that met the diagnostic criteria established by the guidelines for the diagnosis and treatment of sudden deafness, ${ }^{[6]}$ patients with unilateral deafness, onset time $\leq 10$ days, no previous treatment prior admission, and the patients or their family members had given informed consent to this study and signed the consent form. The exclusion criteria were patients with severe heart, lung, liver, kidney, and other diseases, deafness caused by external or inner ear damage, vascular or blood diseases, ototoxic drugs, etc., pregnant or lactating women, contraindications for hyperbaric oxygen therapy, history of allergies or contraindications to related drugs.

\subsection{Treatment}

Both groups were treated with methylcobalamin, vitamin B1, and ginkgo biloba extract injection. The patients in the hyperbaric oxygen treatment group were treated with the YC2475/0.3-101V multi-person hyperbaric oxygen chamber in which the treatment pressure was $0.2 \mathrm{MPa}$ for 20 minutes. Stabilized oxygen inhalation was for 60 minutes, decompression for 20 minutes once a day with each course of treatment lasting 10 days. Then, the curative effects of both groups were compared after 2 courses.

\subsection{Evaluation}

The evaluation criteria of clinical efficacy are as follows. Recover: Hearing at the damaged frequency returns to normal or reaching the level before the disease; Significant effect: Hearing at the damaged frequency improved by $>30 \mathrm{~dB}$ on average; Effective: Hearing at the damaged frequency improved by 15 $30 \mathrm{~dB}$ on average; Ineffective: Hearing at the damaged frequency improved by $<15 \mathrm{~dB}$ on average.

\subsection{Statistical analysis}

The relevant data involved in the research process were analyzed and processed using the Statistical Package for the Social Sciences (SPSS) version 17.0 software. The count data were expressed in percentage $(\%)$ and tested using chi-square $\left(\mathrm{X}^{2}\right) . \mathrm{P}<0.05$ indicated that the difference between the two groups of data was statistically different. 


\section{Results}

Table 1. Comparison of clinical efficacy between the two groups of patients

\begin{tabular}{ccccccc}
\hline Group & $\begin{array}{c}\text { Number } \\
\text { of cases }\end{array}$ & Recover & $\begin{array}{c}\text { Significant } \\
\text { effect }\end{array}$ & Effective & Ineffective & $\begin{array}{c}\text { Effective } \\
\text { rate }\end{array}$ \\
\hline $\begin{array}{c}\text { Conventional } \\
\text { treatment group } \\
\begin{array}{c}\text { Hyperbaric } \\
\text { oxygen treatment } \\
\text { group }\end{array}\end{array}$ & 48 & $13(27.08 \%)$ & $14(29.17 \%)$ & $11(22.92 \%)$ & $10(20.38 \%)$ & $79.17 \%$ \\
\hline
\end{tabular}

As shown in Table 1, comparing the effective rate of treatment in the conventional treatment group (79.17\%), the effective rate of treatment in the hyperbaric oxygen treatment group (93.75\%) was significantly higher and the difference between the two groups was statistically significant $(\mathrm{P}<0.05)$.

\section{Discussion}

The onset of sudden deafness is very unexpected. There are no obvious signs before the onset of the disease. Only a few patients would develop mild colds, fatigue, and other symptoms. Once the disease occurs, the patient's hearing would deteriorate in a few minutes or a few hours. Some patients may have symptoms such as tinnitus or dizziness which would severely affect their daily activities. Therefore, timely treatment is needed to avoid its progression into lifelong deafness. Some scholars believe that sudden deafness is caused by blood clots, edema, spasm, red blood cell aggregation, or other events to the blood vessels in the ears that cause obstruction to the blood circulation, hence leading to hypoxia and necrosis. Among them, red blood cells have a greater impact on the viscosity of blood. Red blood cells promote the aggregation and adhesion of platelets which reduce the elasticity of their membranes that further aggravate the former. These two affect one another causing a vicious cycle resulting in an increase in the resistance of blood flow. This in turn reduces the perfusion in the microcirculation and eventually affects the patient's hearing ${ }^{[7-8]}$. The traditional treatment of sudden deafness focuses on dilating blood vessels, using nutrient cells and energy mixtures, as well as glucocorticoids to resist the immune response in virus infections or disorders. However, the clinical effects from these treatments are not ideal ${ }^{[9-10]}$. Hyperbaric oxygen therapy is a natural therapy that pressurizes air to ensure the chamber is in a high pressure state. During oxygen inhalation, a large amount of oxygen would dissolve in the blood to improve the hypoxic state of tissues. It is often used as an auxiliary treatment for sudden deafness. Hyperbaric oxygen therapy can increase the partial pressure of blood oxygen, improve circulatory disorders and the state of ischemia or hypoxia of the inner ear, reduce edema, as well as improve the therapeutic effect ${ }^{[11-12]}$. Clinical observations have found that drug therapy alone has certain limitations in the recovery of hearing. However, drugs combined with hyperbaric oxygen therapy can quickly improve hearing impairment and shorten the course of the disease especially for patients with severe hearing impairment.

The results of this study showed that the total effective rate of treatment in the hyperbaric oxygen treatment group was higher than that of the conventional treatment group and the difference was statistically significant. This suggests that hyperbaric oxygen treatment can increase blood oxygen content, reduce tissue ischemia and hypoxic damage, promote as well as improve local blood circulation and inner ear microcirculation, accelerate the recovery of damaged tissues, and improve hearing levels. It has significant advantages in hearing recovery. 


\section{Disclosure statement}

The author declares no conflict of interest.

\section{References}

[1] Jiang F, Peng Y, Li Y, et al., 2015, The Effect of Different Administration Methods of Methylprednisolone on Sudden Sensorineural Hearing Loss. Western Medicine, 27(11): 1631-3.

[2] Han Q, Qu X, Liu P, et al., 2015, The Effect of Ginkgo Biloba Extract on the Immune Function of Patients with Sudden Deafness and the Observation of its Curative Effect. Clinical Misdiagnosis and Mistreatment, 28(2): 106-8.

[3] Wan H, 2013, Clinical Observation of Hyperbaric Oxygen in the Treatment of Sudden Deafness. Medical Information, 26(6): 280.

[4] Wang H, Wang S, Weng Q, et al., 2013, The Timing and Efficacy of Hyperbaric Oxygen Therapy for Sudden Deafness. Chinese Journal of Rehabilitation Medicine, 28(7): 648-9.

[5] Wang G, Gao C, 2011, The status quo of hyperbaric oxygen therapy for sudden deafness. Beijing Medicine, 33(1): 51-3.

[6] Editorial Committee of Chinese Journal of Otorhinolaryngology Head and Neck Surgery, Chinese Medical Association Otorhinolaryngology Head and Neck Surgery Branch, 2015, Guidelines for the Diagnosis and Treatment of Sudden Deafness. Chinese Journal of Otorhinolaryngology Head and Neck Surgery, 50(6): 443-7.

[7] Hu L, Tang X, Wu X, 2012, Analysis of the Effect of Hyperbaric Oxygen on Sudden Deafness and Community Health Education. Guide to Chinese Medicine, 10: 690-1.

[8] Wang T, Liu K, Xu H, 2012, Observation on the Efficacy of Nerve Growth Factor Combined with Hyperbaric Oxygen in the Treatment of Sudden Deafness. Chinese Medicine Guide, 10: 171-2.

[9] Li D, Sun L, Han J, 2012, Clinical Observation of Different Types of Sudden Deafness. Contemporary Medicine, 18(3): 49-50.

[10] $\mathrm{Ku} \mathrm{W,} \mathrm{Liu} \mathrm{Y,} \mathrm{Wu} \mathrm{J,} \mathrm{et} \mathrm{al.,} \mathrm{2014,} \mathrm{Meta} \mathrm{Analysis} \mathrm{of} \mathrm{the} \mathrm{Clinical} \mathrm{Efficacy} \mathrm{of} \mathrm{Domestic} \mathrm{Hyperbaric}$ Oxygen Combined with Drugs in the Treatment of Sudden Deafness. Chinese Journal of Otology, 12(1): 93-8.

[11] Bennett MH, Kertesz T, Perleth M, et al., 2012, Hyperbaric Oxygen for Idiopathic Sudden Sensorineural Hearing Loss and Tinnitus. Cochrane Database Systematic Review, 10.

[12] Fan W, Wu Y, Chen X, et al., 2020, Clinical Study of Acupuncture Combined with Hyperbaric Oxygen in the Treatment of Low-Frequency Descending Sudden Deafness. Chinese Journal of Otology, 18(1): 98-102. 